\title{
Retracted: An Analytical Investigation of Fractional-Order Biological Model Using an Innovative Technique
}

\author{
Complexity \\ Received 3 December 2020; Accepted 2 February 2021; Published 23 February 2021 \\ Copyright (C) 2021 Complexity. This is an open access article distributed under the Creative Commons Attribution License, which \\ permits unrestricted use, distribution, and reproduction in any medium, provided the original work is properly cited.
}

Complexity has retracted the article titled "An Analytical Investigation of Fractional-Order Biological Model Using an Innovative Technique" [1] due to substantial similarity to a previous work by other authors [2]. The earlier article was not cited, though another article by those authors was cited in passing as reference [40]. The aim and structure of $[1,2]$ is the same, and many of the equations are nearly identical in notation: examples 2 and 3 in each article are essentially the same and example 4 in [1] is very similar to example 1 in [2], with only example 1 in [1] being original.

The authors did not agree to retraction, arguing that [2] relied on their own earlier article that introduced the Iterative Laplace transform method, citing it and using its equations [3]. They said [2] also relied on another article [4]. Code files for Maple worksheets (.mw files) are available in Supplementary Materials.

\section{Supplementary Materials}

Code files for Maple worksheets. (Supplementary Materials)

\section{References}

[1] H. Khan, A. Khan, M. A. Qurashi, B. Dumitru, and R. Shah, "An Analytical Investigation of Fractional-Order Biological Model Using an Innovative Technique," Complexity, vol. 2020, p. 13, Article ID 5047054, 2020.

[2] S. C. Sharma and R. K. Bairwa, "Exact Solution of Generalized Time-Fractional Biological Population Model By Means of the Iterative Laplace Transform Method," International Journal of Mathematical Archive, vol. 5, no. 12, pp. 40-46, 2014.

[3] H. Jafari, M. Nazari, D. Baleanu, and C. M. Khalique, "A new approach for solving a system of fractional partial differential equations," Computers \& Mathematics with Applications, vol. 66, no. 5, pp. 838-843, 2013.

[4] A. M. A. El-Sayed, S. Z. Rida, and A. A. M. Arafa, "Exact solutions of fractional-order biological population model,"
Communications in Theoretical Physics, vol. 52, no. 6, pp. 992-996, 2009. 


\title{
An Analytical Investigation of Fractional-Order Biological Model Using an Innovative Technique
}

\author{
Hassan Khan (D), Adnan Khan ${ }^{1},{ }^{1}$ Maysaa Al Qurashi, ${ }^{2}$ Dumitru Baleanu, ${ }^{3,4}$ \\ and Rasool Shah $\mathbb{D D}^{1}$ \\ ${ }^{1}$ Department of Mathematics, Abdul Wali Khan University, Mardan, Pakistan \\ ${ }^{2}$ Department of Mathematics, King Saud University, Riyadh 11495, Saudi Arabia \\ ${ }^{3}$ Department of Mathematics, Faculty of Arts and Sciences, Cankaya University, 06530 Ankara, Turkey \\ ${ }^{4}$ Institute of Space Sciences, Magurele-Bucharest, Romania
}

Correspondence should be addressed to Hassan Khan; hassanmath@awkum.edu.pk

Received 4 February 2020; Accepted 18 March 2020; Published 15 April 2020

Academic Editor: Dimitri Volchenkov

Copyright (C) 2020 Hassan Khan et al. This is an open access article distributed under the Creative Commons Attribution License, which permits unrestricted use, distribution, and reproduction in any medium, provided the original work is properly cited.

In this paper, a new so-called iterative Laplace transform method is implemented to investigate the solution of certain important population models of noninteger order. The iterative procedure is combined effectively with Laplace transformation to develop the suggested methodology. The Caputo operator is applied to express the noninteger derivative of fractional order. The series form solution is obtained having components of convergent behavior toward the exact solution. For justification and verification of the present method, some illustrative examples are discussed. The closed contact is observed between the obtained and exact solutions. Moreover, the suggested method has a small volume of calculations; therefore, it can be applied to handle the solutions of various problems with fractional-order derivatives.

\section{Introduction}

Over the last century, fractional differential equations (FDEs) have attracted a great deal of attention from scientists due to their ability to raise real-world issues in numerous engineering fields and physics. FDEs are broadly used in certain fields of science [1-5]. Several phenomena in chemistry, physics, engineering, and other sciences can be effectively described using fractional calculus. Acoustics, the nonlinear oscillation of earthquake, electrochemistry, electromagnetism, signal processing, and diffusion processes can be modeled by fractional equations $[6,7]$. In modern times, it is difficult to imagine the modeling of several real-world issues without the use of fractional partial differential equations (FPDEs). Indeed, this century's calculus [8] can be called a fractional calculus because of the diversity of implementations in different fields of science and technology. The researchers have used several analytical and numerical techniques such as variational iteration method (VIM) [9], homotopy analysis method (HAM) [10], and generalized fractional Taylor series method [11] to solve linear and nonlinear FPDEs.
Any phenomena in the areas of engineering and science may be alternatively modeled via fractional-order derivatives. It is due to their nonlocal properties, which are inherent in some complex structures. They are used as modeling devices in financial, viscoelasticity, transport phenomenon, nanotechnology, control theory, and biological modeling [12-15]. In biology, biological cell membranes are shown to have electrical conductance in a fractional order and are classified among noninteger order systems [16-18]. Anomalous diffusion concepts in nonhomogeneous media can be discussed by noninteger, derivative-based diffusion equations [19-21]. Another example of a fractional-order element is practice, which is a noninteger-ordered electrical circuit with resistance and capacitance properties [10]. In this connection, certain important techniques have been used including the fractional operational matrix method (FOMM) [22, 23], fractional wavelet method (FWM) [24-27], homotopy analysis method (HAM) [28], homotopy perturbation method (HPM) [29], homotopy perturbation transform method (HPTM) [30], Laplace Adomian decomposition 
method (LADM) [31], and fractional variational iteration method (FVIM) [32].

Daftardar-Gejji and Jafari in 2006 have developed the iterative technique to solve nonlinear functional equations $[33,34]$. Later on, the iterative technique is applied to solve noninteger differential equations (DEs) [35]. In recent time, Jafari et al. have used Laplace transform together with an iterative technique for the first time which is nowadays becoming an effective technique named as the iterative Laplace transform method (ILTM) [36]. ILTM is implemented to solve partial differential equations (PDEs) and Fokker-Plank problems [37]. Recently, many other FPDEs have been solved by using ILTM such as time-fractional Schrodinger equations [38], fractional telegraph equations [39], fractional heat and wave-like equations [40], and timefractional Fisher equation [41].

The main theme of the present research work is to use ILTM for obtaining the analytical solution of the noninteger biological population model [42].

$$
\begin{array}{r}
\frac{\partial^{\rho} \psi(\mu, \nu, \tau)}{\partial \tau^{\rho}}=\frac{\partial^{2} \psi^{2}(\mu, \nu, \tau)}{\partial \mu^{2}}+\frac{\partial^{2} \psi^{2}(\mu, \nu, \tau)}{\partial \nu^{2}}+g(\psi(\mu, \nu, \tau)) \\
\tau>0,0<\rho \leq 1,
\end{array}
$$

subject to the starting values: $\psi(\mu, \nu, 0)=g_{0}(\mu, \nu)$.

Equation (1) is identified as the time fractional-order biological population model, used by Gurney and Nisbet as a unique situation for modeling the animal population. In general, movements are made both by mature animals driven by mature invaders or by young animals just reaching maturity moving out of their parental territory to establish their breeding territory. In both cases, the assumption that they would be driven towards neighboring vacant land is much more likely. Hence, movement in this model occurs almost entirely down the gradient of population density and will be faster at high population densities than at low ones, where the population density is represented by $\psi(\mu, \nu, \tau)$ and the population rate is expressed by $g(\psi(\mu, \nu, \tau))$. For $\rho \longrightarrow 1$, Various properties such like Holder estimates for its solution are discussed in $[43,44]$. The three consecutive cases for $g(u)$ are as follows:

$$
\begin{aligned}
& g(\psi)=c \text {, for any constant } c \text { that reduces to Malthusian } \\
& \text { law } \\
& g(\psi)=\psi\left(d_{1}-d_{2} \psi\right) \text {, for positive constants } d_{1} \text { and } d_{2} \\
& \text { that reduce to Verhulst law } \\
& g(\psi)=-d \psi^{k},(d \geq 0,0<k<1) \text {, for positive } d \text { that re- } \\
& \text { duces to porous media }
\end{aligned}
$$

The ILTM solutions are found to be in good contact with the exact solutions of the problems. The solutions at different fractional orders are also calculated. It is investigated that the fractional-order solutions are convergent towards integerorder solution of the problems as fractional order approaches to an integer order. It is also observed that the suggested method is very simple and effective and required small number of calculation. Moreover, the present method provides the series form solution with easily computable components. It is also shown that the series form solution has the desire rate of convergence towards the exact solution of the problem and the closed form solution is achieved.

\section{Definitions and Preliminaries}

In this part of the paper, some important definitions related to FC and Laplace transform are briefly discussed. These preliminaries are important to continue and complete the present research work.

Definition 1. The fractional derivative in terms of Caputo operator is expressed as

$$
\begin{aligned}
D_{\tau}^{\rho} \psi(\mu, \tau) & =\frac{1}{\Gamma(n-\rho)} \int_{0}^{\tau}(\tau-\zeta)^{n-\rho-1} \psi^{(n)}(\mu, \zeta) d \zeta, \\
& n-1<\rho \leq n, n \in N \\
& =J_{\tau}^{n-\rho} D^{n} u(\mu, \tau) .
\end{aligned}
$$

Here,

$$
D^{n}=\frac{d^{n}}{d \tau^{n}}
$$

Definition 2. The fractional integral in terms of Riemann-Liouville integral is expressed as

$$
\begin{array}{r}
j_{\tau}^{\rho} \psi(\mu, \tau)=\frac{1}{\Gamma(\rho)} \int_{0}^{\tau}(\tau-\zeta)^{\rho-1} \psi(\mu, \zeta) d \zeta, \\
\zeta>0,(n-1<\rho \leq n), n \in N,
\end{array}
$$

where $j_{\tau}^{\rho}$ represents the fractional integral operator.

Definition 3. The Laplace transform is describe as

$$
L[g(\tau)]=G(\tau)=\int_{0}^{\infty} e^{-s \tau} g(\tau) d \tau
$$

Definition 4. The Laplace transform of the fractional derivative $D_{\tau}^{\rho} \psi(\mu, \tau)$ is defined as

$$
\begin{array}{r}
L\left[D_{\tau}^{\rho} \psi(\mu, \tau)\right]=s^{\rho} L[\psi(\mu, \tau)]-\sum_{k=0}^{n-1} \psi^{(k)}(\mu, 0) s^{\rho-k-1}, \\
n-1<\rho \leq n, n \in N .
\end{array}
$$

Definition 5. The Mittag-Leffler function is given by

$$
E_{\rho}(z)=\sum_{q=0}^{\infty} \frac{z^{q}}{\Gamma(\rho q+1)}, \quad(\rho \in C, \operatorname{Re}(\rho)>0) .
$$

\section{The Basic Concept of ILTM}

In this section, we will briefly discuss ILTM to solve fractional-order nonlinear PDEs. 


$$
\begin{array}{r}
D_{\tau}^{\rho} \psi(\mu, \nu, \tau)+R \psi(\mu, \nu, \tau)+N \psi(\mu, \nu, \tau)=g(\mu, \nu, \tau), \\
n-1<\rho \leq n, n \in N, \\
\psi^{(k)}(\mu, \nu, 0)=h_{k}(\mu, \nu), \quad k=0,1,2, \ldots, n-1,
\end{array}
$$

where $D_{\tau}^{\rho} \psi(\mu, \nu, \tau)$ is the fractional Caputo operator of order $\rho, n-1<\rho \leq n$, denoted by equation (8), and $R$ and $N$ are linear and nonlinear operators. $g(\mu, \nu, \tau)$ is source function.

Using Laplace transform of equation (8), we get

$$
\begin{aligned}
& L\left[D_{\tau}^{\rho} \psi(\mu, \nu, \tau)\right]+L[R \psi(\mu, \nu, \tau)+N \psi(\mu, \nu, \tau)] \\
& \quad=L[g(\mu, \nu, \tau)] .
\end{aligned}
$$

Applying the property of Laplace differentiation,

$$
\begin{aligned}
L[\psi(\mu, \nu, \tau)]= & \frac{1}{s^{\rho}} \sum_{k=0}^{m-1} s^{\rho-1-k} \psi^{(k)}(\mu, \nu, 0)+\frac{1}{s^{\rho}} L[g(\mu, \nu, \tau)] \\
& -\frac{1}{s^{\rho}} L[R \psi(\mu, \nu, \tau)+N \psi(\mu, \nu, \tau)] .
\end{aligned}
$$
obtain

By using inverse Laplace transform of equation (11), we

$$
\psi(\mu, \nu, \tau)=L^{-1}\left[\frac{1}{s^{\rho}}\left(\sum_{k=0}^{m-1} s^{\rho-1-k} \psi^{k}(\mu, \nu, 0)+L[g(\mu, \nu, \tau)]\right)\right]-L^{-1}\left[\frac{1}{s^{\rho}} L[R \psi(\mu, \nu, \tau)+N \psi(\mu, \nu, \tau)]\right] .
$$

From iterative technique,

$$
\psi(\mu, \nu, \tau)=\sum_{i=0}^{\infty} \psi_{i}(\mu, \nu, \tau)
$$

Since $R$ is a linear operator,

$$
R\left(\sum_{i=0}^{\infty} \psi_{i}(\mu, \nu, \tau)\right)=\sum_{i=0}^{\infty} R\left[\psi_{i}(\mu, \nu, \tau)\right]
$$

$$
\begin{aligned}
N\left(\sum_{i=0}^{\infty} \psi_{i}(\mu, \nu, \tau)\right)= & N\left[\psi_{0}(\mu, \nu, \tau)\right] \\
& +\sum_{i=1}^{\infty}\left\{N \left(\sum_{k=0}^{i} \psi_{k}(\mu, \nu, \tau)\right.\right. \\
& \left.-N\left(\sum_{k=0}^{i-1} \psi_{k}(\mu, \nu, \tau)\right)\right\} .
\end{aligned}
$$

and the nonlinear operator $\mathrm{N}$ is split as

Substituting equations (13)-(15) into equation (12), we obtain

$$
\begin{aligned}
\sum_{i=0}^{\infty} \psi_{i}(\mu, \nu, \tau)= & L^{-1}\left[\frac{1}{s^{\rho}}\left(\sum_{k=0}^{m-1} s^{\rho-1-k} \psi^{k}(\mu, \nu, 0)+L[g(\mu, \nu, \tau)]\right)\right] \\
& -L^{-1}\left[\frac{1}{s^{\rho}} L\left[\sum_{i=0}^{\infty} R\left[\psi_{i}(\mu, \nu, \tau)\right]+N\left[\psi_{0}(\mu, \nu, \tau)\right]+\sum_{i=1}^{\infty}\left\{N\left(\sum_{k=0}^{i} \psi_{k}(\mu, \nu, \tau)-N\left(\sum_{k=0}^{i-1} \psi_{k}(\mu, \nu, \tau)\right)\right)\right\}\right]\right.
\end{aligned}
$$

Using equation (16), we define the following iterative formula:

$$
\begin{aligned}
\psi_{0}(\mu, \nu, \tau) & =L^{-1}\left[\frac{1}{s^{\rho}}\left(\sum_{k=0}^{m-1} s^{\rho-1-k} \psi^{k}(\mu, \nu, 0)+\frac{1}{s^{\rho}} L(g(\mu, \nu, \tau))\right)\right] \\
\psi_{1}(\mu, \nu, \tau) & =-L^{-1}\left[\frac{1}{s^{\rho}} L\left[R\left[\psi_{0}(\mu, \nu, \tau)\right]+N\left[\psi_{0}(\mu, v, \tau)\right]\right],\right. \\
\psi_{m+1}(\mu, \nu, \tau) & =-L^{-1}\left[\frac{1}{s^{\rho}} L\left[R\left(\psi_{m}(\mu, \nu, \tau)\right)-\left\{N\left(\sum_{k=0}^{m} \psi_{k}(\mu, \nu, \tau)\right)-N\left(\sum_{k=0}^{m-1} \psi_{k}(\mu, \nu, \tau)\right)\right\}\right], \quad m \geq 1 .\right.
\end{aligned}
$$

The approximate m-term solution of equations (18) and (19) in form of series is as follows: 


$$
\psi(\mu, \nu, \tau) \cong \psi_{0}(\mu, \nu, \tau)+\psi_{1}(\mu, \nu, \tau)+\psi_{2}(\mu, \nu, \tau)+\cdots+\psi_{m}(\mu, \nu, \tau), \quad m=1,2, \ldots
$$

\section{Implementation of ILTM}

$$
\frac{\partial^{\rho} \psi}{\partial \tau^{\rho}}=\frac{\partial^{2}}{\partial \mu^{2}}\left(\psi^{2}\right)+\frac{\partial^{2}}{\partial \nu^{2}}\left(\psi^{2}\right)+h \psi^{-1}(1-r \psi)
$$

In this section, ILTM is applied to determine the exact solution of some special cases of equation (1). It has been shown that the ILTM is an accurate and appropriate analytical technique to solve nonlinear FPDEs.

Example 1. The biological population model with time noninteger derivative is express as

$$
0<\rho \leq 1, \mu, \nu \in \mathfrak{R}, \tau>0,
$$

with starting values

$$
\psi(\mu, \nu, 0)=\sqrt{\frac{h r}{4} \mu^{2}+\frac{h r}{4} v^{2}+\nu+5}
$$

The Laplace transform to equation (21) is expressed as

$$
\begin{aligned}
s^{\rho} L[\psi(\mu, \nu, \tau)]-\sum_{k=0}^{m-1} \psi^{(k)}(\mu, \nu, 0) s^{\rho-k-1} & =L\left(\frac{\partial^{2}}{\partial \mu^{2}}\left(\psi^{2}\right)+\frac{\partial^{2}}{\partial \nu^{2}}\left(\psi^{2}\right)+h \psi^{-1}(1-r \psi)\right) \\
L[\psi(\mu, \nu, \tau)] & =\frac{1}{s} \sqrt{\frac{h r}{4} \mu^{2}+\frac{h r}{4} v^{2}+\nu+5}+\frac{1}{s^{\rho}}\left[L\left(\frac{\partial^{2}}{\partial \mu^{2}}\left(\psi^{2}\right)+\frac{\partial^{2}}{\partial \nu^{2}}\left(\psi^{2}\right)+h \psi^{-1}(1-r \psi)\right)\right] .
\end{aligned}
$$

Using inverse Laplace transform of equation (24),

$$
\psi(\mu, \nu, \tau)=\sqrt{\frac{h r}{4} \mu^{2}+\frac{h r}{4} \nu^{2}+\nu+5}+L^{-1}\left[\frac{1}{s^{\rho}}\left[L\left(\frac{\partial^{2}}{\partial \mu^{2}}\left(\psi^{2}\right)+\frac{\partial^{2}}{\partial \nu^{2}}\left(\psi^{2}\right)+h \psi^{-1}(1-r \psi)\right)\right]\right]
$$

Using the iterative technique described in equations (14) and (15), we obtain the following solution components of Example 1:

$$
\begin{aligned}
\psi_{0}(\mu, \nu, \tau) & =\sqrt{\frac{h r}{4} \mu^{2}+\frac{h r}{4} \nu^{2}+v+5} \\
\psi_{1}(\mu, \nu, \tau) & =L^{-1}\left[\frac{1}{s^{\rho}}\left[L\left(\frac{\partial^{2}}{\partial \mu^{2}}\left(\psi_{0}^{2}\right)+\frac{\partial^{2}}{\partial \nu^{2}}\left(\psi_{0}^{2}\right)+h \psi_{0}^{-1}\left(1-r \psi_{0}\right)\right)\right]\right] \\
& =h\left(\left(\frac{h r}{4} \mu^{2}+\frac{h r}{4} v^{2}+\nu+5\right)^{-1 / 2}\right) \frac{\tau^{\rho}}{\Gamma(\rho+1)}, \\
\psi_{2}(\mu, \nu, \tau) & =L^{-1}\left[\frac{1}{s^{\rho}}\left[L\left(\frac{\partial^{2}}{\partial \mu^{2}}\left(\psi_{1}^{2}\right)+\frac{\partial^{2}}{\partial \nu^{2}}\left(\psi_{1}^{2}\right)+h \psi_{1}^{-1}\left(1-r \psi_{1}\right)\right)\right]\right] \\
& =-2 h^{2}\left(\left(\frac{h r}{4} \mu^{2}+\frac{h r}{4} v^{2}+\nu+5\right)^{-3 / 2}\right) \frac{\tau^{2 \rho}}{\Gamma(2 \rho+1)}, \\
\psi_{3}(\mu, \nu, \tau) & =L^{-1}\left[\frac{1}{s^{\rho}}\left[L\left(\frac{\partial^{2}}{\partial \mu^{2}}\left(\psi_{2}^{2}\right)+\frac{\partial^{2}}{\partial \nu^{2}}\left(\psi_{2}^{2}\right)+h \psi_{2}^{-1}\left(1-r \psi_{2}\right)\right)\right]\right] \\
& =3 h^{3}\left(\left(\frac{h r}{4} \mu^{2}+\frac{h r}{4} v^{2}+\nu+5\right)^{-5 / 2}\right) \frac{\tau^{3 \rho}}{\Gamma(3 \rho+1)} .
\end{aligned}
$$


The series form of analytical solution is given as

$$
\begin{aligned}
\psi(\mu, \nu, \tau)= & \psi_{0}(\mu, \nu, \tau)+\psi_{1}(\mu, \nu, \tau)+\psi_{2}(\mu, \nu, \tau)+\psi_{3}(\mu, \nu, \tau)+\cdots \\
= & \left(\frac{h r}{4} \mu^{2}+\frac{h r}{4} v^{2}+\nu+5\right)^{1 / 2}+h\left(\left(\frac{h r}{4} \mu^{2}+\frac{h r}{4} v^{2}+\nu+5\right)^{-1 / 2}\right) \frac{t^{\rho}}{\Gamma(\rho+1)} \\
& -2 h^{2}\left(\left(\frac{h r}{4} \mu^{2}+\frac{h r}{4} v^{2}+v+5\right)^{-3 / 2}\right) \frac{\tau^{2 \rho}}{\Gamma(2 \rho+1)} \\
& \left.+3 h^{3}\left(\frac{h r}{4} \mu^{2}+\frac{h r}{4} v^{2}+v+5\right)^{-5 / 2}\right) \frac{\tau^{3 \rho}}{\Gamma(3 \rho+1)}+\cdots \\
\psi(\mu, \nu, \tau)= & \psi_{0}+\frac{h \tau^{\rho}}{\psi_{0}} \sum_{n=0}^{\infty} \frac{n+1}{\Gamma((n+1) \rho+1)}\left(\frac{-h \tau^{\rho}}{\psi_{0}^{2}}\right)^{n}
\end{aligned}
$$

The exact result is given by

$$
\psi(\mu, \nu, \tau)=\sqrt{\frac{h r}{4} \mu^{2}+\frac{h r}{4} \nu^{2}+v+2 h \tau+5} .
$$

$$
\frac{\partial^{\rho} \psi}{\partial \tau^{\rho}}=\frac{\partial^{2}}{\partial \mu^{2}}\left(\psi^{2}\right)+\frac{\partial^{2}}{\partial v^{2}}\left(\psi^{2}\right)+h \psi
$$

with initial condition

$$
\psi(\mu, \nu, 0)=\sqrt{\mu \nu} .
$$

Example 2. The biological population model with time noninteger derivative is expressed as

The Laplace transform to equation (29) is expressed as

$$
\begin{aligned}
s^{\rho} L[\psi(\mu, \nu, \tau)]-\sum_{k=0}^{m-1} \psi^{(k)}(\mu, \nu, 0) s^{\rho-k-1} & =L\left(\frac{\partial^{2}}{\partial \mu^{2}}\left(\psi^{2}\right)+\frac{\partial^{2}}{\partial \nu^{2}}\left(\psi^{2}\right)+h \psi\right), \\
s^{\rho} L[\psi(\mu, \nu, \tau)] & =\psi^{(0)}(\mu, \nu, 0) \frac{s^{\rho}}{s}+L\left(\frac{\partial^{2}}{\partial \mu^{2}}\left(\psi^{2}\right)+\frac{\partial^{2}}{\partial \nu^{2}}\left(\psi^{2}\right)+h \psi\right), \\
L[\psi(\mu, \nu, \tau)] & =\frac{1}{s} \sqrt{\mu \nu}+\frac{1}{s^{\rho}}\left[L\left(\frac{\partial^{2}}{\partial \mu^{2}}\left(\psi^{2}\right)+\frac{\partial^{2}}{\partial \nu^{2}}\left(\psi^{2}\right)+h \psi\right)\right] .
\end{aligned}
$$

Using inverse Laplace transform of equation (32), $\psi(\mu, \nu, \tau)=\sqrt{\mu \nu}+L^{-1}\left[\frac{1}{s^{\rho}}\left[L\left(\frac{\partial^{2}}{\partial \mu^{2}}\left(\psi^{2}\right)+\frac{\partial^{2}}{\partial \nu^{2}}\left(\psi^{2}\right)+h \psi\right)\right]\right]$.
Using the iterative technique described in equations (14) and (15), we obtain the following solution components of Example 2: 


$$
\begin{aligned}
\psi_{0}(\mu, \nu, \tau) & =\sqrt{\mu \nu} \\
\psi_{1}(\mu, \nu, \tau) & =L^{-1}\left[\frac{1}{s^{\rho}}\left[L\left(\frac{\partial^{2}}{\partial \mu^{2}}\left(\psi_{0}^{2}\right)+\frac{\partial^{2}}{\partial \nu^{2}}\left(\psi_{0}^{2}\right)+h \psi_{0}\right)\right]\right] \\
& =h \sqrt{\mu \nu} \frac{\tau^{\rho}}{\Gamma(\rho+1)}, \\
\psi_{2}(\mu, v, \tau) & =L^{-1}\left[\frac{1}{s^{\rho}}\left[L\left(\frac{\partial^{2}}{\partial \mu^{2}}\left(\psi_{1}^{2}\right)+\frac{\partial^{2}}{\partial \nu^{2}}\left(\psi_{1}^{2}\right)+h \psi_{1}\right)\right]\right] \\
& =h^{2} \sqrt{\mu \nu} \frac{\tau^{2 \rho}}{\Gamma(2 \rho+1)}, \\
\psi_{3}(\mu, \nu, \tau) & =L^{-1}\left[\frac{1}{s^{\rho}}\left[L\left(\frac{\partial^{2}}{\partial \mu^{2}}\left(\psi_{2}^{2}\right)+\frac{\partial^{2}}{\partial \nu^{2}}\left(\psi_{2}^{2}\right)+h \psi_{2}\right)\right]\right] \\
& =h^{3} \sqrt{\mu \nu} \frac{\tau^{3 \rho}}{\Gamma 3 \rho+1} .
\end{aligned}
$$

The series form of analytical solution is given as

$$
\begin{gathered}
\psi(\mu, \nu, \tau)=\psi_{0}(\mu, \nu, \tau)+\psi_{1}(\mu, \nu, \tau)+\psi_{2}(\mu, \nu, \tau)+\psi_{3}(\mu, \nu, \tau)+\cdots \\
=\sqrt{\mu \nu}+h \sqrt{\mu \nu} \frac{\tau^{\rho}}{\Gamma(\rho+1)}+h^{2} \sqrt{\mu \nu} \frac{\tau^{2 \rho}}{\Gamma(2 \rho+1)}+h^{3} \sqrt{\mu \nu} \frac{\tau^{3 \rho}}{\Gamma(3 \rho+1)}+\cdots \\
\psi(\mu, \nu, \tau)=\sqrt{\mu \nu} \sum_{k=0}^{\infty} \frac{\left(h \tau^{\rho}\right)^{k}}{\Gamma(k \rho+1)}
\end{gathered}
$$

The exact solution is given by

$$
\psi(\mu, \nu, \tau)=\sqrt{\mu \nu} E_{\rho}\left(h \tau^{\rho}\right)
$$

where $E_{\rho}\left(h \tau^{\rho}\right)$ is the Mittag-Leffler function defined as

$$
E_{\rho}(z)=\sum_{k=0}^{\infty} \frac{z^{k}}{\Gamma(\rho k+1)} .
$$

As $\rho \longrightarrow 1$, we have

$$
\begin{aligned}
\psi(\mu, \nu, \tau)=\sqrt{\mu \nu} \sum_{k=0}^{\infty} \frac{(h \tau)^{k}}{k !}=\sqrt{\mu \nu} e^{h \tau} & \text { The Laplace transform to equation (40) } \\
s^{\rho} L[\psi(\mu, \nu, \tau)]-\sum_{k=0}^{m-1} \psi^{(k)}(\mu, \nu, 0) s^{\rho-k-1} & =L\left(\frac{\partial^{2}}{\partial \mu^{2}}\left(\psi^{2}\right)+\frac{\partial^{2}}{\partial \nu^{2}}\left(\psi^{2}\right)+\psi\right), \\
s^{\rho} L[\psi(\mu, \nu, \tau)] & =\psi^{(0)}(\mu, \nu, 0) \frac{s^{\rho}}{s}+L\left(\frac{\partial^{2}}{\partial \mu^{2}}\left(\psi^{2}\right)+\frac{\partial^{2}}{\partial \nu^{2}}\left(\psi^{2}\right)+\psi\right) \\
L[\psi(\mu, \nu, \tau)] & =\frac{1}{s} \sqrt{\mu \nu}+\frac{1}{s^{\rho}}\left[L\left(\frac{\partial^{2}}{\partial \mu^{2}}\left(\psi^{2}\right)+\frac{\partial^{2}}{\partial \nu^{2}}\left(\psi^{2}\right)+\psi\right)\right]
\end{aligned}
$$


Using inverse Laplace transform of equation (43), we obtain $\psi(\mu, \nu, \tau)=\sqrt{\mu \nu}+L^{-1}\left[\frac{1}{s^{\rho}}\left[L\left(\frac{\partial^{2}}{\partial \mu^{2}}\left(\psi^{2}\right)+\frac{\partial^{2}}{\partial \nu^{2}}\left(\psi^{2}\right)+\psi\right)\right]\right]$.
Using the iterative technique described in equations (14) and (15), we obtain the following solution components of Example 3:

$$
\begin{aligned}
\psi_{0}(\mu, \nu, \tau) & =\sqrt{\sin \mu \sinh v} \\
\psi_{1}(\mu, \nu, \tau) & =L^{-1}\left[\frac{1}{s^{\rho}}\left[L\left(\frac{\partial^{2}}{\partial \mu^{2}}\left(\psi_{0}^{2}\right)+\frac{\partial^{2}}{\partial \nu^{2}}\left(\psi_{0}^{2}\right)+\psi_{0}\right)\right]\right] \\
& =\sqrt{\sin \mu \sinh v} \frac{\tau^{\rho}}{\Gamma(\rho+1)}, \\
\psi_{2}(\mu, \nu, \tau) & =L^{-1}\left[\frac{1}{s^{\rho}}\left[L\left(\frac{\partial^{2}}{\partial \mu^{2}}\left(\psi_{1}^{2}\right)+\frac{\partial^{2}}{\partial v^{2}}\left(\psi_{1}^{2}\right)+\psi_{1}\right)\right]\right] \\
& =\sqrt{\sin \mu \sinh v} \frac{\tau^{2 \rho}}{\Gamma(2 \rho+1)}, \\
\psi_{3}(\mu, \nu, \tau) & =L^{-1}\left[\frac{1}{s^{\rho}}\left[L\left(\frac{\partial^{2}}{\partial \mu^{2}}\left(\psi_{2}^{2}\right)+\frac{\partial^{2}}{\partial \nu^{2}}\left(\psi_{2}^{2}\right)+\psi_{2}\right)\right]\right] \\
& =\sqrt{\sin \mu \sinh \nu} \frac{\tau^{3 \rho}}{\Gamma(3 \rho+1)} .
\end{aligned}
$$

The series form of analytical solution is given as

$$
\begin{aligned}
& \begin{aligned}
\psi(\mu, \nu, \tau) & =\psi_{0}(\mu, \nu, \tau)+\psi_{1}(\mu, \nu, \tau)+\psi_{2}(\mu, \nu, \tau)+\psi_{3}(\mu, \nu, \tau)+\cdots \\
& =\sqrt{\sin \mu \sinh v}+\sqrt{\sin \mu \sinh v} \frac{\tau^{\rho}}{\Gamma(\rho+1)}+\sqrt{\sin \mu \sinh v} \frac{\tau^{2 \rho}}{\Gamma(2 \rho+1)}+\sqrt{\sin \mu \sinh v} \frac{\tau^{3 \rho}}{\Gamma(3 \rho+1)}+\cdots
\end{aligned} \\
& \psi(\mu, \nu, \tau)=\sqrt{\sin \mu \sinh v} \sum_{k=0}^{\infty} \frac{\tau^{k \rho}}{\Gamma(\rho k+1)},
\end{aligned}
$$

The exact result is given by

$$
\psi(\mu, \nu, \tau)=\sqrt{\sin \mu \sinh v} E_{\rho}\left(h \tau^{\rho}\right) .
$$

As $\rho \longrightarrow 1$, we have

$\psi(\mu, \nu, \tau)=\sqrt{\sin \mu \sinh \nu} \sum_{k=0}^{\infty} \frac{(\tau)^{k}}{k !}=\sqrt{\sin \mu \sinh v} e^{\tau}$.
Example 4. The biological population model with time noninteger derivative is expressed as

$$
\frac{\partial^{\rho} \psi}{\partial \tau^{\rho}}=\frac{\partial^{2}}{\partial \mu^{2}}\left(\psi^{2}\right)+\frac{\partial^{2}}{\partial \nu^{2}}\left(\psi^{2}\right)+\psi(1-r \psi)
$$

with initial condition

$$
\psi(\mu, \nu, 0)=\exp ^{1 / 2 \sqrt{r / 2}(\mu+\nu)} .
$$


The Laplace transform to equation (49) is expressed as

$$
\begin{aligned}
s^{\rho} L[\psi(\mu, \nu, \tau)]-\sum_{k=0}^{m-1} \psi^{(k)}(\mu, \nu, 0) s^{\rho-k-1} & =L\left(\frac{\partial^{2}}{\partial \mu^{2}}\left(\psi^{2}\right)+\frac{\partial^{2}}{\partial \nu^{2}}\left(\psi^{2}\right)+\psi(1-r \psi)\right), \\
s^{\rho} L[\psi(\mu, \nu, \tau)] & =\psi^{(0)}(\mu, \nu, 0) \frac{s^{\rho}}{s}+L\left(\frac{\partial^{2}}{\partial \mu^{2}}\left(\psi^{2}\right)+\frac{\partial^{2}}{\partial \nu^{2}}\left(\psi^{2}\right)+\psi(1-r \psi)\right), \\
L[\psi(\mu, \nu, \tau)] & =\frac{1}{s} \exp ^{1 / 2 \sqrt{r / 2}(\mu+\nu)}+\frac{1}{s^{\rho}}\left[L\left(\frac{\partial^{2}}{\partial \mu^{2}}\left(\psi^{2}\right)+\frac{\partial^{2}}{\partial \nu^{2}}\left(\psi^{2}\right)+\psi(1-r \psi)\right)\right] .
\end{aligned}
$$

Using inverse Laplace transform of equation (52),

$$
\psi(\mu, \nu, \tau)=\exp ^{1 / 2 \sqrt{r / 2}(\mu+\nu)}+L^{-1}\left[\frac{1}{\mathcal{s}^{\rho}}\left[L\left(\frac{\partial^{2}}{\partial \mu^{2}}\left(\psi^{2}\right)+\frac{\partial^{2}}{\partial \nu^{2}}\left(\psi^{2}\right)+\psi(1-r \psi)\right)\right]\right]
$$

Using the iterative technique described in equations (14) and (15), we obtain the following solution components of Example 4:

$$
\begin{aligned}
\psi_{0}(\mu, \nu, \tau) & =\exp ^{1 / 2 \sqrt{r / 2}(\mu+v)} \\
\psi_{1}(\mu, \nu, \tau) & =L^{-1}\left[\frac{1}{s^{\rho}}\left[L\left(\frac{\partial^{2}}{\partial \mu^{2}}\left(\psi_{0}^{2}\right)+\frac{\partial^{2}}{\partial \nu^{2}}\left(\psi_{0}^{2}\right)+\psi_{0}\left(1-r \psi_{0}\right)\right)\right]\right] \\
& =\exp ^{1 / 2 \sqrt{r / 2}(\mu+v)} \frac{\tau^{\rho}}{\Gamma(\rho+1)}, \\
\psi_{2}(\mu, \nu, \tau) & =L^{-1}\left[\frac{1}{s^{\rho}}\left[L\left(\frac{\partial^{2}}{\partial \mu^{2}}\left(\psi_{1}^{2}\right)+\frac{\partial^{2}}{\partial v^{2}}\left(\psi_{1}^{2}\right)+\psi_{1}\left(1-r \psi_{1}\right)\right)\right]\right] \\
& =\exp ^{1 / 2 \sqrt{r / 2}(\mu+v)} \frac{\tau^{2 \rho}}{\Gamma(2 \rho+1)}, \\
\psi_{3}(\mu, \nu, \tau) & =L^{-1}\left[\frac{1}{s^{\rho}}\left[L\left(\frac{\partial^{2}}{\partial \mu^{2}}\left(\psi_{2}^{2}\right)+\frac{\partial^{2}}{\partial \nu^{2}}\left(\psi_{2}^{2}\right)+\psi_{2}\left(1-r \psi_{2}\right)\right)\right]\right] \\
& =\exp ^{1 / 2 \sqrt{r / 2}(\mu+v)} \frac{\tau^{3 \rho}}{\Gamma(3 \rho+1)} .
\end{aligned}
$$




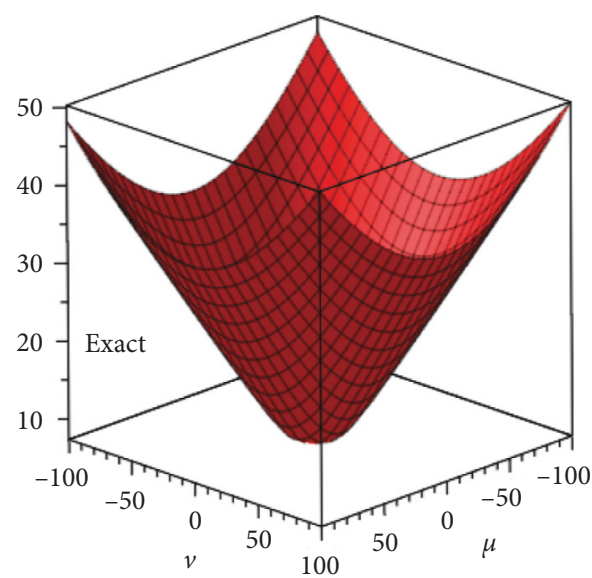

(a)

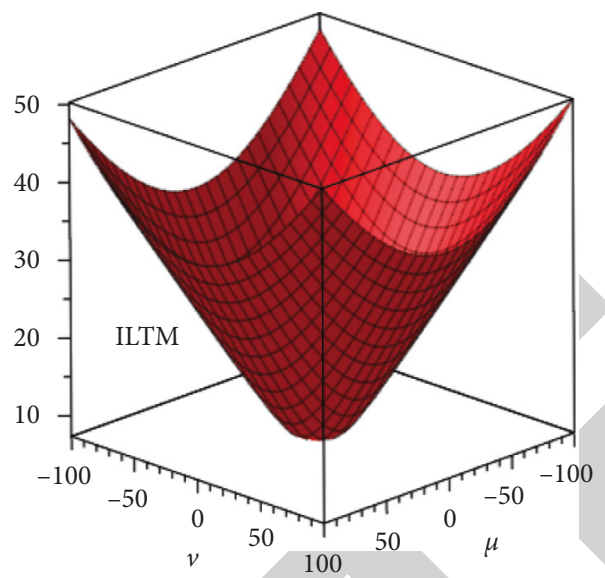

(b)

Figure 1: The solution plot of Example 1: (a) exact solution and (b) ILTM solution at $\rho=1, h=0.01$, and $r=48$.

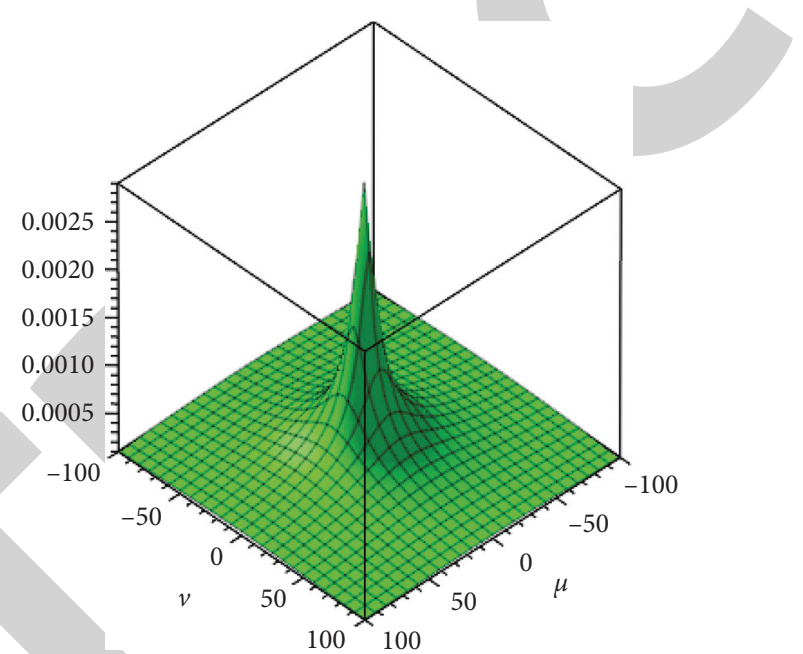

Figure 2: The absolute error for Example 1 at $\rho=1$.

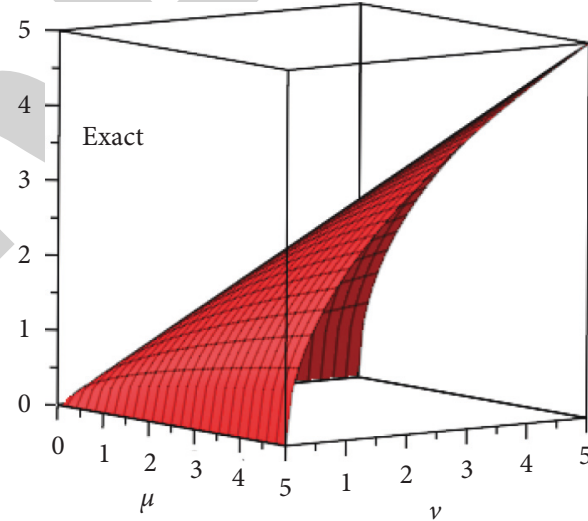

(a)

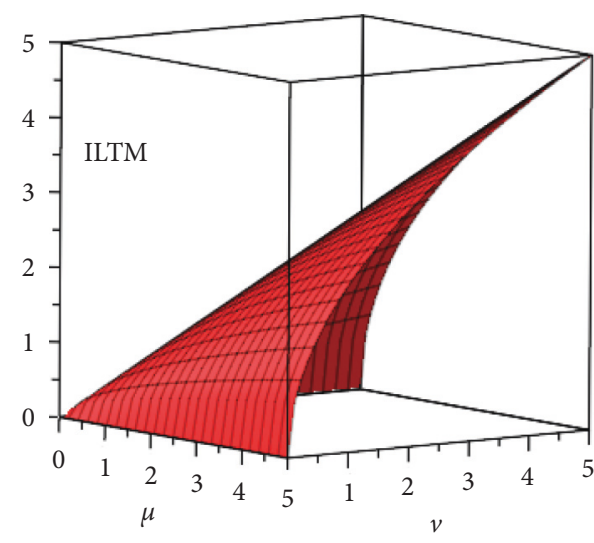

(b)

FIGURE 3: Solution graph of Example 2: (a) exact solution and (b) ILTM solution at $\rho=1, h=0.01$, and $r=48$. 


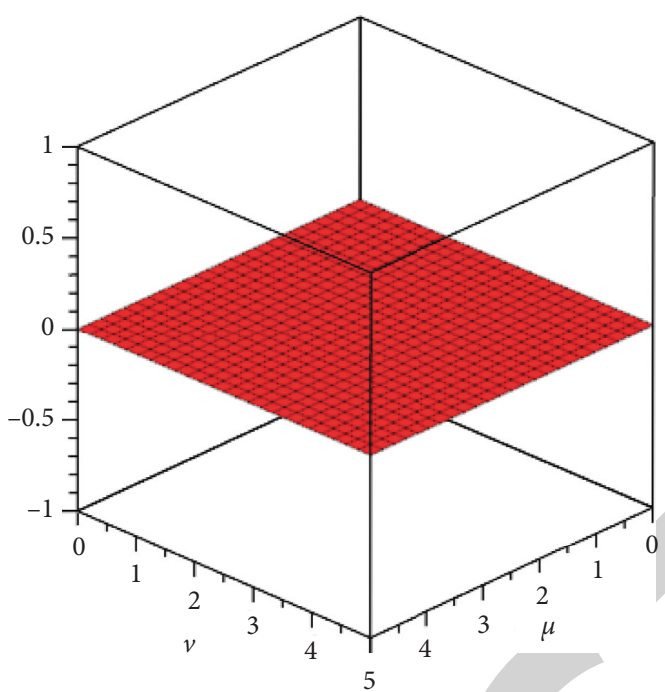

FIgURE 4: The absolute error for Example 2 at $\rho=1$

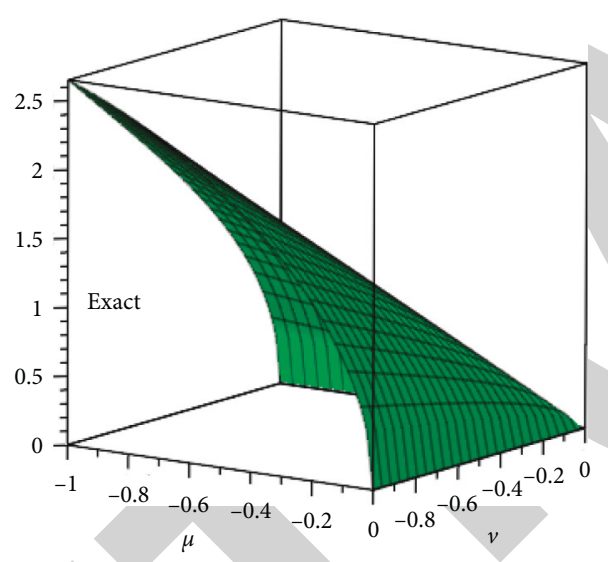

(a)

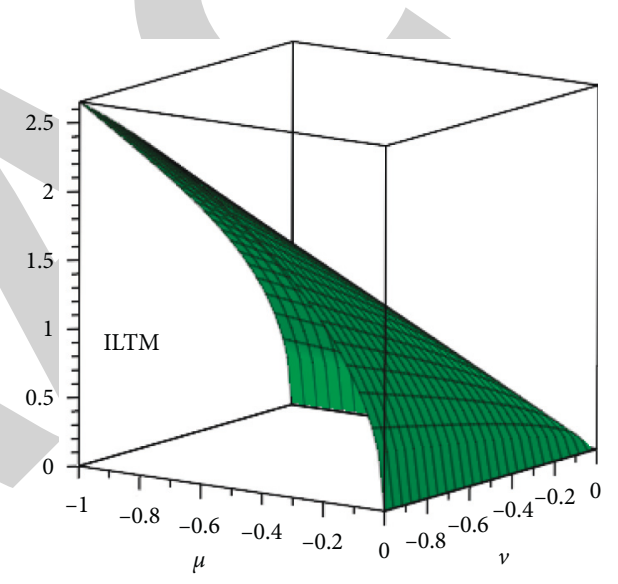

(b)

FIgUre 5: The solution plot of Example 3: (a) exact solution and (b) ILTM solution at $\rho=1, h=0.01$, and $r=48$.
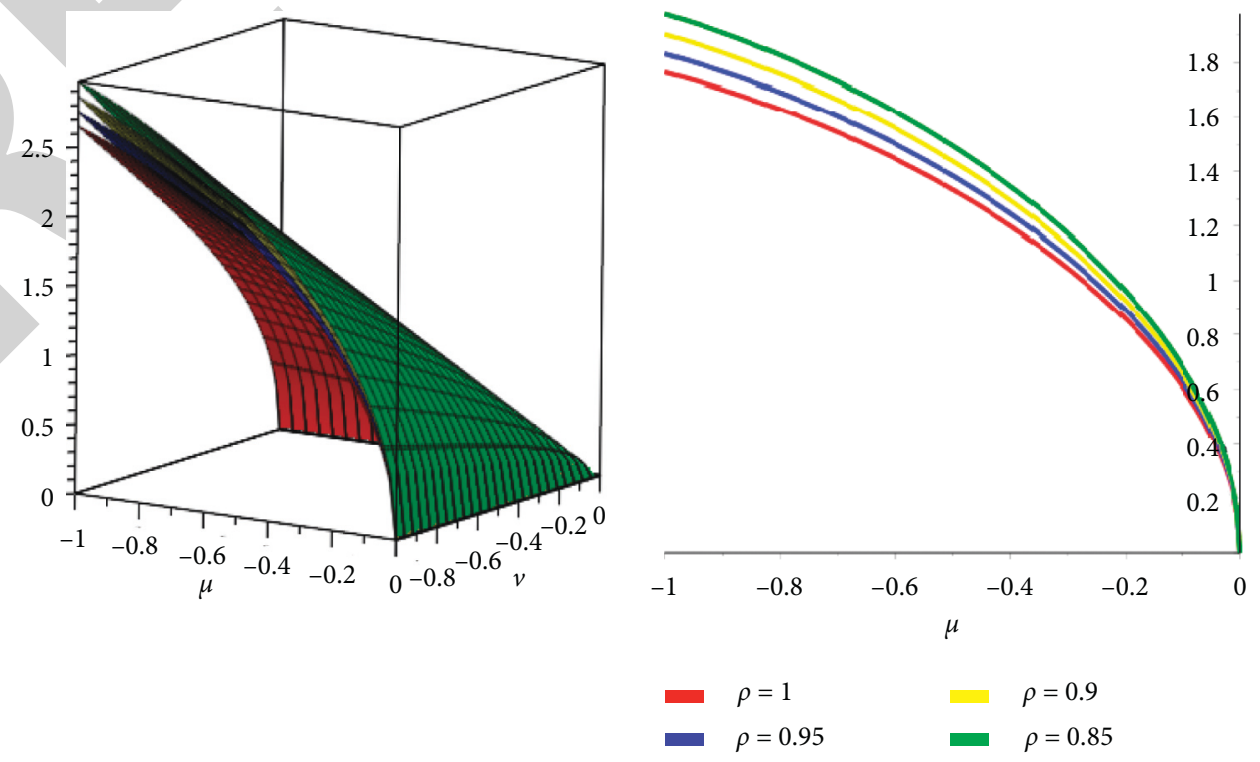

(a)

(b)

FIGURE 6: Different fractional orders of $\rho$ in Example 3 


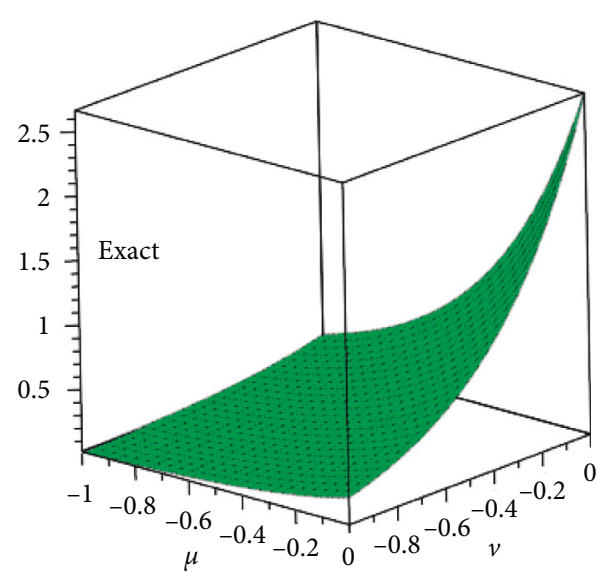

(a)

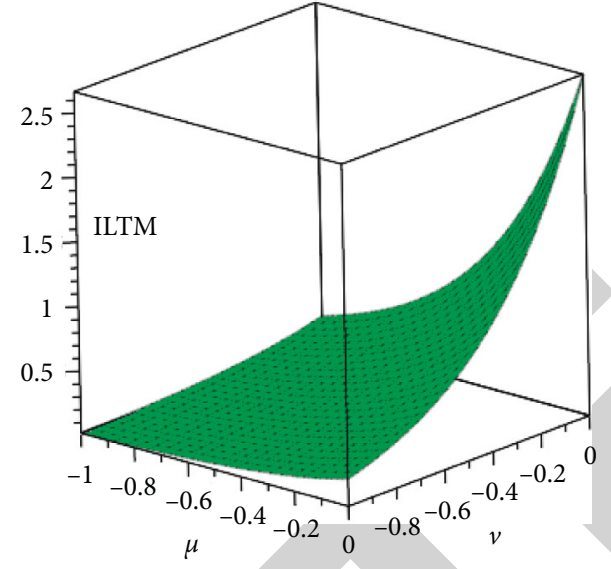

(b)

FIgURE 7: The solution plot of Example 4: (a) exact solution and (b) ILTM solution at $\rho=1, h=0.01$, and $r=48$.

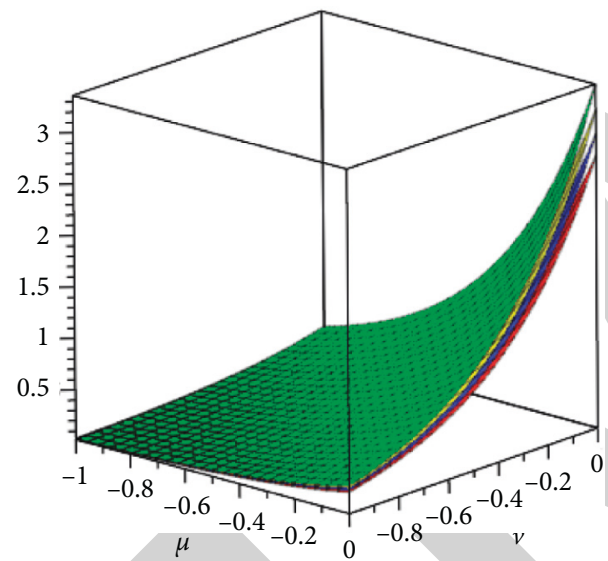

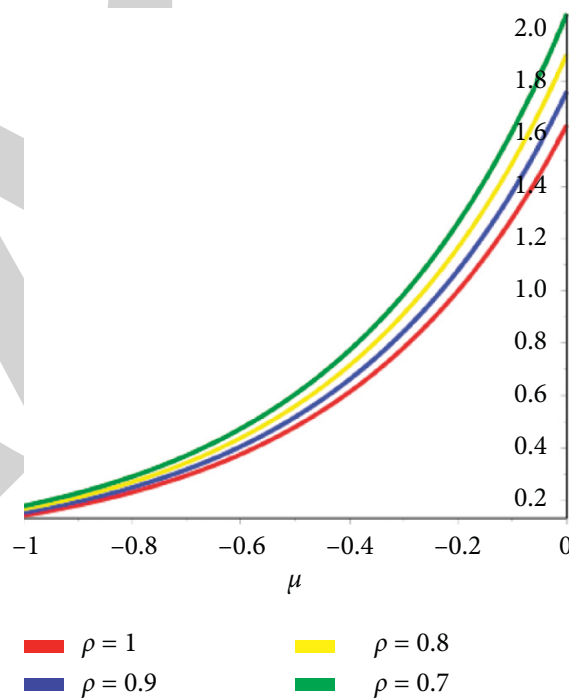

(b)

Figure 8: Different fractional orders of $\rho$ in Example 4.

The series form of analytical solution is given as

$$
\begin{aligned}
\psi(\mu, \nu, \tau)= & \psi_{0}(\mu, \nu, \tau)+\psi_{1}(\mu, \nu, \tau)+\psi_{2}(\mu, \nu, \tau) \\
& +\psi_{3}(\mu, \nu, \tau)+\cdots \\
= & \exp ^{1 / 2 \sqrt{r / 2}(\mu+v)}+\exp ^{1 / 2 \sqrt{r / 2}(\mu+\nu)} \frac{t^{\rho}}{\Gamma(\rho+1)} \\
& +\exp ^{1 / 2 \sqrt{r / 2}(\mu+\nu)} \frac{\tau^{2 \rho}}{\Gamma(2 \rho+1)}+\exp ^{1 / 2 \sqrt{r / 2}(\mu+v)} \\
& \cdot \frac{\tau^{3 \rho}}{\Gamma(3 \rho+1)}+\cdots, \\
\psi(\mu, \nu, \tau)= & \exp ^{1 / 2 \sqrt{r / 2}(\mu+\nu)} \sum_{k=0}^{\infty} \frac{\tau^{k \rho}}{\Gamma(\rho k+1)} .
\end{aligned}
$$

The exact result is given by

$$
\psi(\mu, \nu, \tau)=\exp ^{1 / 2 \sqrt{r / 2}(\mu+\nu)} E_{\rho}\left(h \tau^{\rho}\right)
$$

As $\rho \longrightarrow 1$, we have

$$
\begin{aligned}
\psi(\mu, \nu, \tau) & =\exp ^{1 / 2 \sqrt{r / 2}(\mu+\nu)} \sum_{k=0}^{\infty} \frac{(\tau)^{k}}{k !} \\
& =\exp ^{1 / 2 \sqrt{r / 2}(\mu+\nu)+\tau} .
\end{aligned}
$$

\section{Review Results}

In the present work, ILTM is used to solve some important biological models of noninteger order. The solution of the suggested technique is to explain with the help of its graphical representation. Figure 1 shows the solution graphs of exact and ILTM for Example 1 at $\rho=1$. It is verified that 
the ILTM solution is closely related to the exact solution. In Figure 2, the error analysis of ILTM for Example 1 is discussed. It is observed that the considered technique has an accuracy of a sufficient degree. Similarly, in Figure 3, the solution plot of ILTM and exact solution is shown for Example 2. These solution graphs are very close and confirmed the reliability of the suggested method. Moreover, the higher degree of accuracy is achieved as represented by Figure 4. The exact and ILTM results of Example 3 are compared in Figure 5. The solution for both exact solution and ILTM solution is identical and supports the reliability of the suggested method. In Figure 6, the solution at various fractional orders of Example 3 is calculated. It is investigated that as fractional order approaches to an integer order, fractional-order solutions are convergent to integer-order solutions. In Figure 7, the same graphical representation has been made for the exact and ILTM solution of Example 4. Figure 7 provides the graphical layout of the solution of Example 4 at different fractional orders. The convergence phenomena of the solutions at different fractional orders can be seen in Figure 8.

\section{Conclusion}

The present research article is related to solve fractionalorder biological population models, using an efficient analytical technique. The current method is applied for fractional and integer-order models. The solution graphs for ILTM and exact solutions to the problems are plotted. It is investigated that the ILTM results are in strong agreement within the actual solutions of the current technique. The ILTM solutions of the problems at different fractional orders are also shown with the help of their graphical representation. The phenomena of convergence fractional-order solutions toward the integer-order solution are observed. This behavior of the obtained solution has confirmed the efficiency of the suggested scheme. Due to an effective and straight forward implementation, the suggested method can be modified for the solution of other FPDEs arising in applied sciences.

\section{Data Availability}

The numerical data used to support the findings of this study are included within the article.

\section{Conflicts of Interest}

The authors declare that there are no conflicts of interest regarding the publication of this article.

\section{Acknowledgments}

This study was supported by the Department of Mathematics, King Saud University, Riyadh 11495, Saudi Arabia.

\section{References}

[1] R. Shah, H. Khan, D. Baleanu, P. Kumam, and M. Arif, “A novel method for the analytical solution of fractional
Zakharov-Kuznetsov equations," Advances in Difference Equations, vol. 2019, no. 1, pp. 1-14, 2019.

[2] R. Shah, H. Khan, U. Farooq, D. Baleanu, P. Kumam, and M. Arif, "A new analytical technique to solve system of fractional-order partial differential equations," IEEE Access, vol. 7, pp. 150037-150050, 2019.

[3] M. Yavuz and N. Özdemir, "Comparing the new fractional derivative operators involving exponential and Mittag-Leffler kernel," Discrete \& Continuous Dynamical Systems-S, vol. 13, no. 3, pp. 995-1006, 2020.

[4] E. Ucar, N. Özdemir, and E. Altun, "Fractional order model of immune cells influenced by cancer cells," Mathematical Modelling of Natural Phenomena, vol. 14, no. 3, p. 308, 2019.

[5] F. Evirgen, S. Ucar, N. Özdemir, and Z. Hammouch, "System response of an alcoholism model under the effect of immigration via non-singular kernel derivative," Discrete and Continuous Dynamical Systems-S, 2018.

[6] S. Uçar, E. Uçar, N. Özdemir, and Z. Hammouch, "Mathematical analysis and numerical simulation for a smoking model with Atangana-Baleanu derivative," Chaos, Solitons \& Fractals, vol. 118, pp. 300-306, 2019.

[7] M. Modanli, "On the numerical solution for third order fractional partial differential equation by difference scheme method," An International Journal of Optimization and Control: Theories andApplications (IJOCTA), vol. 9, no. 3, pp. 1-5, 2019.

[8] Y. Cenesiz, O. Tasbozan, and A. Kurt, "Functional variable method for conformable fractional modified KdV-ZK equation and Maccari system," Tbilisi Mathematical Journal, vol. 10, no. 1, pp. 118-126, 2017.

[9] A. M. Wazwaz, "The variational iteration method for solving linear and nonlinear ODEs and scientific models with variable coefficients," Central European Journal of Engineering, vol. 4, no. 1, pp. 64-71, 2014.

[10] S. Liao, Homotopy Analysis Method in Nonlinear Differential Equations, pp. 153-165, Higher Education Press, Beijing, China, 2012.

[11] I. Jaradat, M. Alquran, and K. Al-Khaled, "An analytical study of physical models with inherited temporal and spatial memory," The European Physical Journal Plus, vol. 133, no. 4, p. 162, 2018.

[12] D. Baleanu, K. Diethelm, E. Scalas, and J. J. Trujillo, Fractional Calculus, Vol. 3 of Series on Complexity. Nonlinearity and Chaos, World Scientific, Singapore, 2012.

[13] M. A. Imran, "Application of fractal fractional derivative of power law kernel $\left({ }^{\mathrm{FFP}} 0 D_{x}^{\alpha, \beta}\right)$ to $\mathrm{MHD}$ viscous fluid flow between two plates," Chaos, Solitons \& Fractals, vol. 134, Article ID 109691, 2020.

[14] M. Aleem, M. I. Asjad, A. Shaheen, and I. Khan, "MHD influence on different water based nanofluids $\left(\mathrm{TiO}_{2}, \mathrm{Al}_{2} \mathrm{O}_{3}\right.$, $\mathrm{CuO}$ ) in porous medium with chemical reaction and Newtonian heating," Chaos, Solitons \& Fractals, vol. 130, Article ID 109437, 2020.

[15] M. Asjad, "Fractional mechanism with power law (singular) and exponential (non-singular) kernels and its applications in bio heat transfer model," International Journal of Heat and Technology, vol. 37, no. 3, pp. 846-852, 2019.

[16] M. Aleem, M. Imran Asjad, M. S. R. Chowdhury, and A. Hussanan, "Analysis of mathematical model of fractional viscous fluid through a vertical rectangular channel," Chinese Journal of Physics, vol. 61, pp. 336-350, 2019.

[17] B. Wang, M. Tahir, M. Imran, M. Javaid, and C. Y. Jung, "Semi analytical solutions for fractional Oldroyd-B fluid through rotating annulus," IEEE Access, vol. 7, pp. 72482-72491, 2019. 
[18] K. S. Cole, "Electric conductance of biological systems," Cold Spring Harbor Symposia on Quantitative Biology, vol. 1, pp. 107-116, 1933.

[19] W. G. Glöckle and T. F. Nonnenmacher, "A fractional calculus approach to self-similar protein dynamics," Biophysical Journal, vol. 68, no. 1, pp. 46-53, 1995.

[20] M. A. Bayrak and A. Demir, "A new approach for space-time fractional partial differential equations by residual power series method," Applied Mathematics and Computation, vol. 336, pp. 215-230, 2018.

[21] A. Demir, M. A. Bayrak, and E. Ozbilge, "A new approach for the approximate analytical solution of space-time fractional differential equations by the homotopy analysis method," Advances in Mathematical Physics, vol. 2019, no. 12, Article ID 5602565, 2019.

[22] V. E. Arkhincheev, "Anomalous diffusion in inhomogeneous media: some exact results," Modelling Measurement and Control a General Physics Electronics and Electrical Engineering, vol. 49, p. 11, 1993.

[23] B. T. Krishna, "Studies on fractional order differentiators and integrators: a survey," Signal Processing, vol. 91, no. 3, pp. 386-426, 2011.

[24] Y. Li and N. Sun, "Numerical solution of fractional differential equations using the generalized block pulse operational matrix," Computers \& Mathematics with Applications, vol. 62, no. 3, pp. 1046-1054, 2011.

[25] A. Saadatmandi and M. Dehghan, "A new operational matrix for solving fractional-order differential equations," Computers \& Mathematics with Applications, vol. 59, no. 3, pp. 13261336, 2010.

[26] R. Shah, U. Farooq, H. Khan, D. Baleanu, P. Kumam, and M. Arif, "Fractional view analysis of third order KortewegeDe Vries equations, using a new analytical technique," Frontiers in Physics, vol. 7, p. 244, 2020.

[27] Y. Li, "Solving a nonlinear fractional differential equation using Chebyshev wavelets," Communications in Nonlinear Science and Numerical Simulation, vol. 15, no. 9, pp. 22842292, 2010.

[28] Ü. Lepik, "Solving fractional integral equations by the Haar wavelet method," Applied Mathematics and Computation, vol. 214, no. 2, pp. 468-478, 2009.

[29] N. A. Khan, N.-U. Khan, A. Ara, and M. Jamil, "Approximate analytical solutions of fractional reaction-diffusion equations," Journal of King Saud University-Science, vol. 24, no. 2, pp. 111-118, 2012.

[30] X. Zhang and L. Juan, "An analytic study on time-fractional Fisher equation using homotopy perturbation method," Walailak Journal of Science and Technology (WJST), vol. 11, no. 11, pp. 975-985, 2014.

[31] Y. Khan and Q. Wu, "Homotopy perturbation transform method for nonlinear equations using He's polynomials," Computers \& Mathematics with Applications, vol. 61, no. 8, pp. 1963-1967, 2011.

[32] N. H. Sweilam, M. M. Khader, and R. F. Al-Bar, "Numerical studies for a multi-order fractional differential equation," Physics Letters A, vol. 371, no. 1-2, pp. 26-33, 2007.

[33] S. Das, "Analytical solution of a fractional diffusion equation by variational iteration method," Computers \& Mathematics with Applications, vol. 57, no. 3, pp. 483-487, 2009.

[34] I. Ali, H. Khan, R. Shah, D. Baleanu, P. Kumam, and M. Arif, "Fractional view analysis of acoustic wave equations, using fractional-order differential equations," Applied Sciences, vol. 10, no. 2, p. 610, 2020.
[35] H. Jafari, Iterative methods for solving system of fractional differential equations, Ph.D. thesis, Pune University, Pune, India, 2006.

[36] S. Bhalekar and V. Daftardar-Gejji, "Solving evolution equations using a new iterative method," Numerical Methods for Partial Differential Equations: An International Journal, vol. 26, no. 4, pp. 906-916, 2010.

[37] H. Jafari, M. Nazari, D. Baleanu, and C. M. Khalique, "A new approach for solving a system of fractional partial differential equations," Computers \& Mathematics with Applications, vol. 66 , no. 5, pp. 838-843, 2013.

[38] L. Yan, "Numerical solutions of fractional Fokker-Planck equations using iterative Laplace transform method," Abstract and Applied Analysis, vol. 2013, Article ID 465160, 7 pages, 2013.

[39] H. Khan, R. Shah, M. Arif, and S. Bushnaq, "The Chebyshev wavelet method (CWM) for the numerical solution of fractional HIV infection of CD4+T cells model," International Journal of Applied and Computational Mathematics, vol. 6, no. 2, pp. 1-17, 2020.

[40] S. C. Sharma and R. K. Bairwa, "A reliable treatment of iterative Laplace transform method for fractional telegraph equations," Annals of Pure and Applied Mathematics, vol. 9, no. 1, pp. 81-89, 2015.

[41] R. Shah, H. Khan, P. Kumam, and M. Arif, “An analytical technique to solve the system of nonlinear fractional partial differential equations," Mathematics, vol. 7, no. 6, p. 505, 2019.

[42] H. Khan, R. Shah, P. Kumam, and M. Arif, "Analytical solutions of fractional-order heat and wave equations by the natural transform decomposition method," Entropy, vol. 21, no. 6 , p. 597, 2019.

[43] A. M. A. El-Sayed, S. Z. Rida, and A. A. M. Arafa, "Exact solutions of fractional-order biological population model," Communications in Theoretical Physics, vol. 52, no. 6, pp. 992-996, 2009.

[44] Y.-G. Lu, "Hölder estimates of solutions of biological population equations," Applied Mathematics Letters, vol. 13, no. 6, pp. 123-126, 2000. 